11,12

\title{
Особенности вырождения основного состояния спин-псевдоспиновой модели двумерного магнетика вблизи точки фрустрации
}

\author{
() Д.Н. Ясинская, В.А. Улитко, Ю.Д. Панов \\ Уральский федеральный университет, \\ Екатеринбург, Россия \\ E-mail: daria.iasinskaia@urfu.ru
}

Поступила в Редакцию 9 апреля 2021 г.

В окончательной редакции 9 апреля 2021 г.

Принята к публикации 19 апреля 2021 г.

С помощью классического метода Монте-Карло исследуются свойства основного состояния и фазовых переходов спин-псевдоспиновой модели, описывающей двумерный изинговский магнетик с конкурирующими зарядовым и спиновым взаимодействиями. Эта конкуренция приводит к вырождению основного состояния и фрустрации системы. Показано, что вырождение основного состояния наблюдается в области фрустрации, где вероятности обнаружения двух разных упорядоченных состояний отличны от нуля. На основе гистограммного анализа данных проведен анализ типа фазовых переходов. Установлено, что вблизи точки фрустрации наблюдаются фазовые переходы первого рода в зависимости от соотношения между спиновым $(s=1 / 2)$ и псевдоспиновым $(S=1)$ взаимодействиями.

Ключевые слова: разбавленный изинговский магнетик, фрустрация, метод Монте-Карло, основное состояние, фазовые переходы.

DOI: 10.21883/FTT.2021.09.51311.05H

\section{1. Введение}

Задача выяснения микроскопической природы необычных свойств систем с сильными межчастичными взаимодействиями становится более сложной при наличии конкуренции и/или сосуществования различных типов упорядочений. Эта проблема актуальна для высокотемпературных сверхпроводящих (ВТСП) купратов, где в нормальном состоянии сосуществуют статический магнитный порядок и волны зарядовой плотности [1]. Для описания систем с различными типами степеней свободы широко используются псевдоспиновые модели [2,3].

Рассматриваемая в настоящей работе спин-псевдоспино вая модель была введена нами ранее [4] для изучения конкуренции зарядовой (связанной с псевдоспином) и спиновой степеней свободы в квазидвумерном ВТСП-купрате в нормальном состоянии. Как и многие другие псевдоспиновые модели, эта модель также подходит для описания физических свойств разбавленных фрустрированных магнетиков. Благодаря гибкости в определении физического смысла псевдоспиновых операторов, псевдоспиновые модели активно используются для описания свойств самых разных физических систем. Примером может служить известная модель Блюм-Эмери-Гриффитса [5], которая используется для описания свойств бинарных сплавов [6], многокомпонентных жидкостей и разбавленных магнетиков [7], а также холодных атомов и сверхпроводящих систем $[8,9]$. В зависимости от физического смысла псевдоспинов, даже простые решеточные модели вроде моделей Изинга или Поттса могут описывать большой класс реальных физических систем [10].
Конкуренция между зарядовым (псевдоспиновым) и магнитным (спиновым) упорядочениями в исследуемой модели приводит к фрустрации системы. Системы с двумя или более конкурирующими взаимодействиями, каждое из которых приводит к определенному виду упорядочения, обладают большим разнообразием упорядоченных фазовых состояний с различными термодинамическими свойствами и со сложной симметрией. Также фрустрированные системы обладают высокой чувствительностью к внешним воздействиям, полям, анизотропии, примесям $[10,11]$ и демонстрируют изменения в критическом поведении [12]. Кроме того, модели фрустрированных магнетиков интересны своей тесной связью со спиновыми жидкостями, стеклами [13] и льдами [14].

\section{2. Модель и методы исследования}

Спин-псевдоспиновая модель [4] представляет собой систему смешанного типа со спином $s=1 / 2$ и псевдоспином $S=1$. Псевдоспин используется нами для описания валентных состояний плоскостей $\mathrm{CuO}_{2}$ ВТСПкупратов типа $\mathrm{La}_{2-x} \mathrm{Sr}_{x} \mathrm{CuO}_{4}$ в рамках псевдоспинового формализма. Здесь псевдоспин $S=1$ представляет собой „зарядовый триплет“ связанный с тремя устойчивыми валентными состояниями центров $\left[\mathrm{CuO}_{4}\right]^{5-, 6-, 7-}$ в $\mathrm{CuO}_{2}$-плоскости. Проекции псевдоспина $S_{z}= \pm 1$ связаны с двумя немагнитными центрами $\left[\mathrm{CuO}_{4}\right]^{5-, 7-}$ со спином 0 в основном состоянии. Магнитное состояние $\left[\mathrm{CuO}_{4}\right]^{6-}$ связано с проекцией псевдоспина $S_{z}=0$ и является спиновым дублетом $s=1 / 2$. Таким образом, каждый узел двумерной решетки может находиться в двух 
зарядовых состояниях, связанных с двумя проекциями псевдоспина $S_{z}= \pm 1$, и в двух спиновых состояниях, связанных с двумя проекциями спина $s_{z}= \pm 1 / 2$.

Гамильтониан спин-псевдоспиновой модели магнетика включает в себя одноионную псевдоспиновую анизотропию $\Delta$, псевдоспиновое изинговское обменное взаимодействие $V$, а также обычное спиновое обменное взаимодействие в форме Изинга $J$ :

$$
H=\Delta \sum_{i} S_{i z}^{2}+V \sum_{\langle i j\rangle} S_{i z} S_{j z}+\tilde{J} \sum_{\langle i j\rangle} \sigma_{i z} \sigma_{j z}-\mu \sum_{i} S_{i z}
$$

Суммирование идет по $N$ узлам двумерной квадратной решетки, $\langle i j\rangle$ означает ближайших соседей. Здесь $\sigma_{i z}=P_{0 i} s_{i z} / s-$ нормированная компонента спина $s=1 / 2$, умноженная на проекционный оператор $P_{0 i}=1-S_{i z}^{2}$, выделяющий магнитное $\left[\mathrm{CuO}_{4}\right]^{6-}$-состояние с $S_{z}=0, \tilde{J}=J s^{2}=J / 4, \mu-$ химический потенциал, необходимый для учета условия постоянства заряда:

$$
n=\frac{1}{N} \sum_{i} S_{i z}=\text { const, }
$$

где $n-$ плотность заряда, отсчитываемого от заряда центра $\left[\mathrm{CuO}_{4}\right]^{6-}$.

„Взаимодействие“ и конкуренция между зарядовым и спиновым упорядочениями в данной модели обусловлены кинематическим ограничением, связанным с условием полноты набора возможных состояний на данном узле решетки: спинового дублета с $S_{z}=0$ и $s_{z}= \pm 1 / 2$ и зарядового дублета с $S_{z}= \pm 1$ и $s=0$. В явном виде в гамильтониане (1) эта связь учтена во входящем в $\sigma_{z}$ проекционном операторе $P_{0}$, где он играет роль оператора спиновой плотности для центров $\left[\mathrm{CuO}_{4}\right]^{6-}$.

Отметим также, что к выражению (1) в приближении точечных зарядов может быть приведено зарядзарядовое взаимодействие $\hat{V}$ достаточно общего вида. Используя проекторы на различные валентные состояния $\mathrm{CuO}_{4}$-центра, $P_{1}$ для $\left[\mathrm{CuO}_{4}\right]^{5-}, P_{0}$ для $\left[\mathrm{CuO}_{4}\right]^{6-}$ и $P_{-1}$ для $\left[\mathrm{CuO}_{4}\right]^{7-}$, запишем

$$
\hat{V}=\sum_{\langle i j\rangle} \sum_{a, b} V_{a b} P_{i a} P_{j b} .
$$

Предполагая, что для ближайших соседей на квадратной решетке $V_{a b}=V q_{a} q_{b}$, где $q_{0}$ - заряд центра $\left[\mathrm{CuO}_{4}\right]^{6-}$, $q_{1}=q_{0}+1$ и $q_{-1}=q_{0}-1$ - заряды центров $\left[\mathrm{CuO}_{4}\right]^{5-}$ и $\left[\mathrm{CuO}_{4}\right]^{7-}$, соответственно, и учитывая тождество $S_{i z}=P_{i 1}-P_{i,-1}$, получим выражение

$$
\hat{V}=V \sum_{\langle i j\rangle} S_{i z} S_{j z}+4 V q_{0} \sum_{i} S_{i z}+2 N V q_{0}^{2},
$$

которое отличается от (1) только сдвигом уровней отсчета энергии и химического потенциала.

Таким образом, система может быть разбавлена заряженными примесями, которые являются подвижными и способны вступать в заряд-зарядовое взаимодействие. Свойства системы в основном состоянии были изучены нами ранее в приближении среднего поля [15]. Далее, в приближении среднего поля [16] и в приближении Бете [17] были получены температурные фазовые диаграммы и термодинамические характеристики. Внесение в модель зарядового беспорядка также может существенно влиять на критическое поведение и фазовые состояния спиновых систем $[18,19]$. Однако в настоящей работе целью является изучение влияния конкуренции двух взаимодействий на формирование основного состояния изинговского магнетика, поэтому рассматривается только случай $n=0$.

Для численного моделирования спин-псевдоспиновой системы мы использовали классический метод МонтеКарло (МК). Обычный алгоритм Метрополиса был модифицирован [20] с целью возможности фиксации плотности заряда (2) на каждом шаге МК, а также был распараллелен с применением технологии CUDA. Расчеты МК проводились на квадратной решетке с периодическими граничными условиями, линейными размерами $L a$, количеством узлов $N=L \times L$, где $a-$ постоянная решетки, принятая далее за 1 . Все расчеты производились одновременно для ста копий системы с целью уточнения результатов, а также для анализа поведения системы вблизи фрустрации.

Температурные зависимости удельной теплоемкости и (псевдо)магнитной восприимчивости определяются с помощью флуктуационных соотношений:

$$
\begin{aligned}
& C=\left(\frac{\partial E}{\partial T}\right)_{H}=\frac{1}{N} \frac{\left\langle E^{2}\right\rangle-\langle E\rangle^{2}}{k_{b} T^{2}}, \\
& \chi=\left(\frac{\partial O}{\partial H}\right)_{T}=N \frac{\left\langle O^{2}\right\rangle-\langle O\rangle^{2}}{k_{b} T},
\end{aligned}
$$

где $k_{b}$ - постоянная Больцмана, $E$ - энергия системы с гамильтонианом (1). Параметр порядка $O$ для шахматных антиферромагнитной $(m)$ и зарядовоупорядоченной $(M)$ фаз характеризует спонтанную ориентационную упорядоченность системы (псевдо)спинов и определяется следующим образом:

$$
O=\left\{\begin{array}{l}
m=m_{1}-m_{2} \\
M=M_{1}-M_{2}
\end{array}\right.
$$

Здесь $m_{\lambda}=\frac{1}{N} \sum_{i \in \lambda} s_{i z}-$ намагниченность подрешетки $\lambda=1,2, M_{\lambda}=\frac{1}{N} \sum_{i \in \lambda} S_{i z}-$ средний заряд подрешетки $\lambda$ (псевдонамагниченность).

Критические температуры фазовых переходов (ФП) определялись по максимумам теплоемкости и восприимчивостей, а также проверялись с помощью метода кумулянтов Биндера [21].

\section{3. Результаты и их обсуждение}

На рис. 1 представлены температурные фазовые диаграммы в зависимости от параметра одноионной 


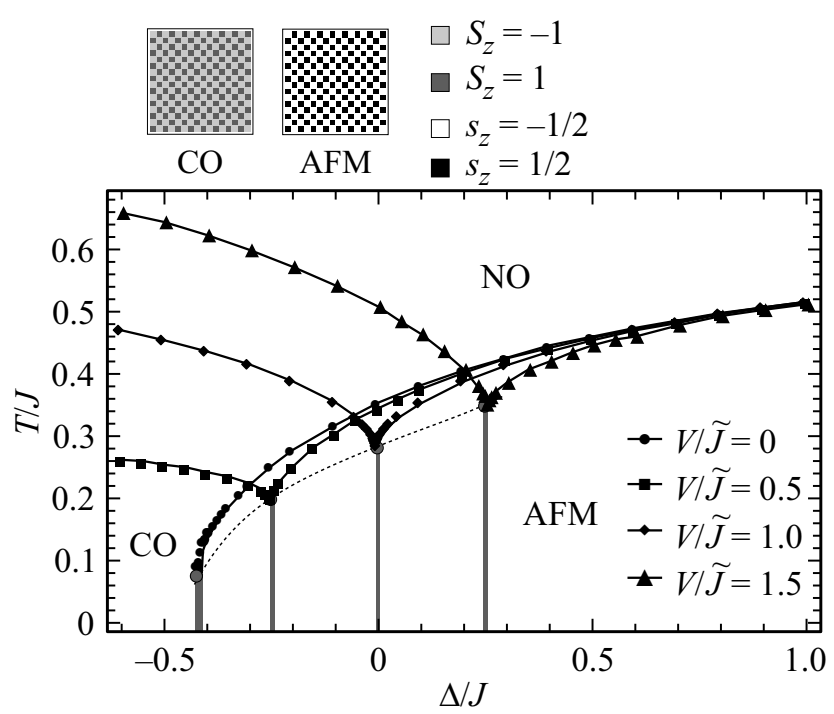

Pис. 1. Температурные фазовые диаграммы, полученные в отсутствие допированного заряда $(n=0)$, для $V / \tilde{J}=0$, $V / \tilde{J}=0.5, V / \tilde{J}=1, V / \tilde{J}=1.5$. Серыми точками обозначено положение точки фрустрации $\Delta^{*}$. Серые вертикальные полосы соответствуют областям фрустрации для каждого соотношения $V / \tilde{J}$.

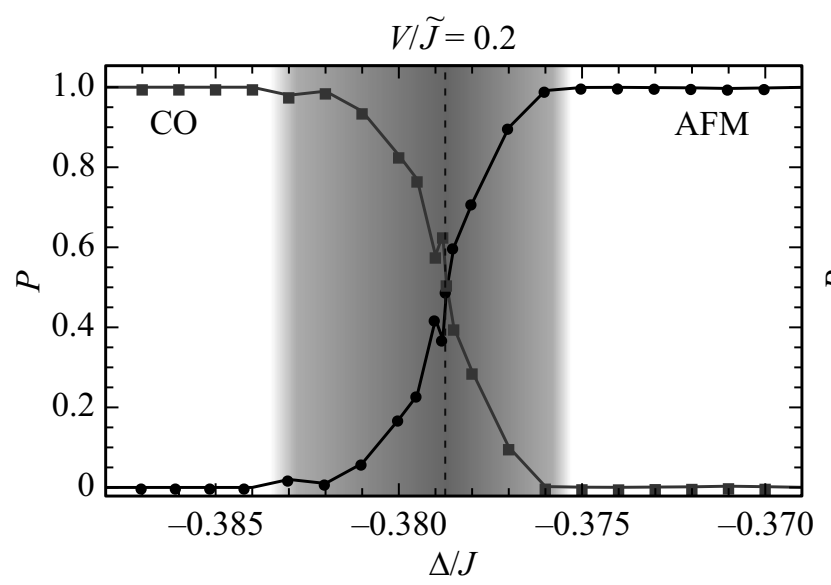

$V / \widetilde{J}=1.0$

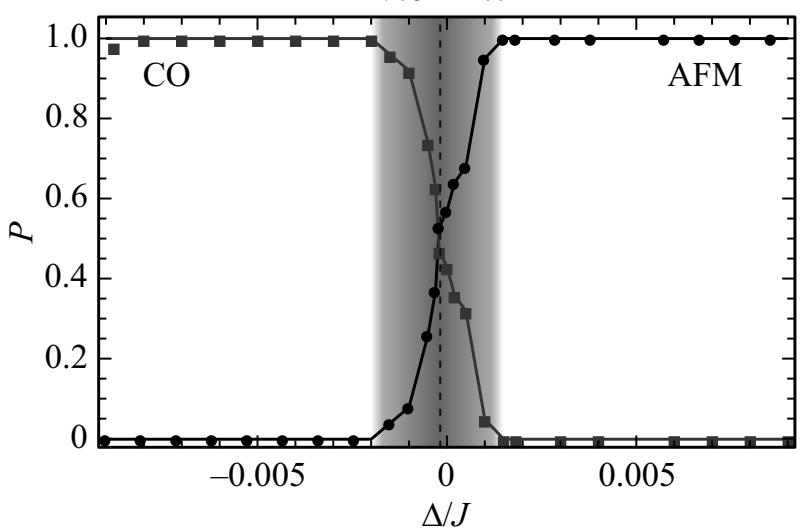

анизотропии $\Delta / J$ для различных соотношений между обменными взаимодействиями $V / \tilde{J}$. Фазовые диаграммы имеют характерный вид „галочек“, в правой части которых система переходит из неупорядоченного (NO, nonordered) парамагнитного состояния в антиферромагнитно упорядоченное (АFM) состояние спинов $s=1 / 2$, тогда как в левой - в шахматный зарядовый порядок (CO) псевдоспинов $S=1$ (кроме случая $V / \tilde{J}=0$, когда зарядовый порядок невозможен). На границе этих двух фаз из-за конкуренции между зарядовым и магнитным упорядочениями основное состояние системы оказывается вырождено. Параметр одноузельной анизотропии $\Delta / J$ в данной системе является фрустрирующим, а точка, в которой происходит смена типа упорядочения в основном состоянии, определяет точку фрустрации $\Delta^{*}$. Эта точка является классическим аналогом квантовой критической точки. Такой тип фазовых диаграмм фрустрированных систем довольно распространен в литературе, и, например, представлен моделью Изинга с конкурирующими взаимодействиями между ближайшими и вторыми соседями на ОЦК- или ПК-решетке [22,23]. Однако для модели со смешанными спином $s=1$ и псевдоспином $S=1$ такие фазовые диаграммы были получены впервые.
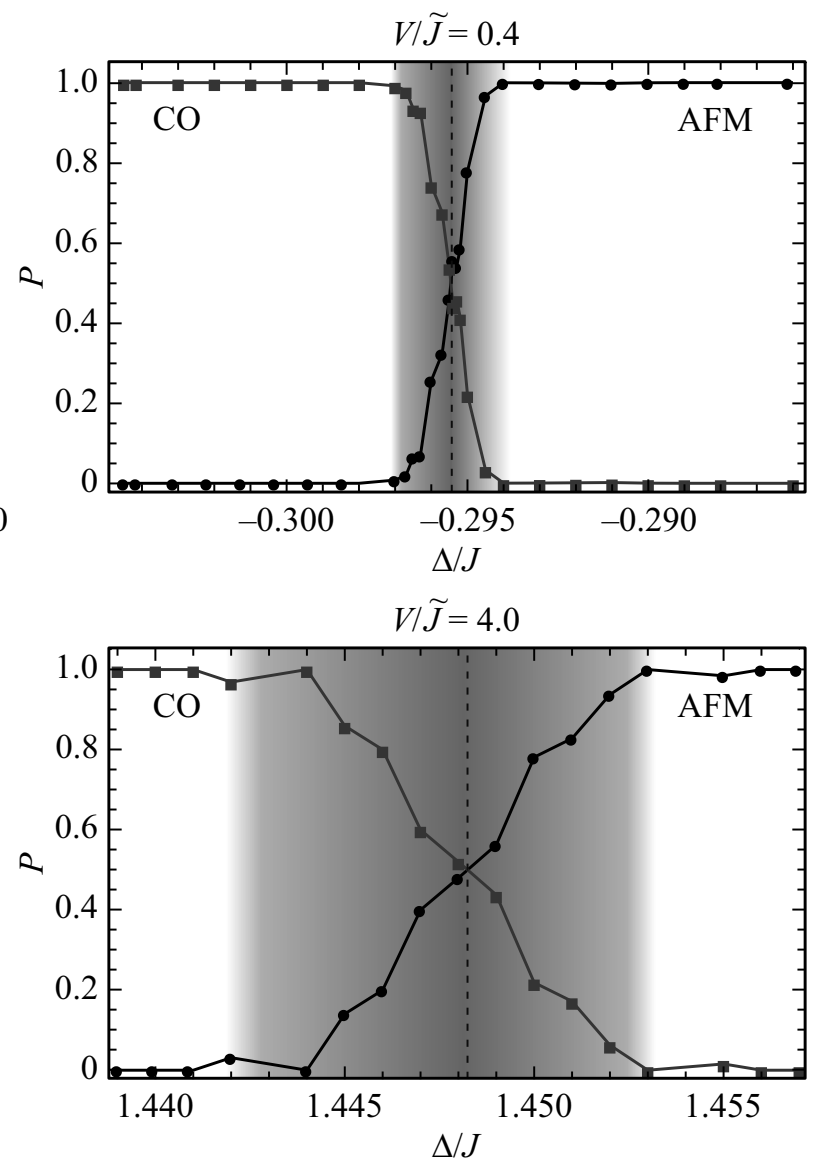

Рис. 2. Распределения вероятностей формирования AFM- (черная кривая) и СО- (серая кривая) типов упорядочений в основном состоянии вблизи точки фрустрации для $V / \tilde{J}=0.2, V / \tilde{J}=0.4, V / \tilde{J}=1, V / \tilde{J}=4$. Серым показаны области, в которых вероятность обнаружения обеих фаз в основном состоянии отлична от нуля. 
При изменении соотношения между обменными взаимодействиями точка фрустрации $\Delta^{*}$ смещается в сторону увеличения $\Delta / J$, и при $V / \tilde{J}=1$ оказывается равной нулю, а температурная фазовая диаграмма становится симметричной относительно линии $\Delta^{*}=0$. Таким образом, имеется разграничение между случаями сильного $(V \leq \tilde{J})$ и слабого $(V>\tilde{J})$ спиновых обменов. Это разграничение приводит к качественным различиям между $\Delta / J-n$-диаграммами основного состояния, а также к другим эффектам, проявляющимся при увеличении плотности фиксированного заряда $n$, как описано в работах $[15,19]$.

Предполагается, что такого рода фазовые диаграммы обладают лишь одной точкой фрустрации, соответствующей минимуму зависимостей температур ФП от фрустрирующего параметра. Однако нами было обнаружено, что основное состояние системы вырождено не в одной конкретной точке, а в целой „области фрустрации“. В этой области и зарядовое, и антиферромагнитное упорядочения формируются в основном состоянии с отличными от нуля вероятностями. Это значит, что при идентичных модельных параметрах и условиях термализации часть копий системы упорядочивается антиферромагнитно, а часть - зарядово. Эти области обозначены серыми вертикальными линиями на рис. 1.

Зависимости вероятностей формирования СО- и AFMтипов упорядочений для разных соотношений $V / \tilde{J}$ от фрустрирующего параметра $\Delta / J$ представлены на рис. 2. Расчеты были выполнены для 100 копий системы с линейными размерами решеток $L=128$, а также проверены для $L=192$. Вдали от точки фрустрации основное состояние системы точно определено: формируется либо СО-, либо АFМ-порядок. Однако при приближении к точке фрустрации энергии этих фаз становятся одинаковыми, и в отдельных копиях системы может формироваться как CO-, так и AFM-фаза. Таким образом, точкой фрустрации будет являться значение параметра $\Delta / J$, в котором обе фазы в основном состоянии равновероятны. Областью фрустрации мы будем называть область вокруг точки фрустрации, в которой вероятности формирования обеих фаз отличны от нуля (серые области на рис. 1,2).

Конкуренция зарядового и спинового взаимодействий приводит не только к вырождению основного состояния, но и к изменению типа ФП. На рис. 3 показаны температурные зависимости параметров порядка AFM $(m$, черные кривые) и $\mathrm{CO}(M$, серые кривые) для нескольких различных копий системы, полученных при одинаковых параметрах $L=128, V / \tilde{J}=4, \Delta / J=1.455$. При этом зависимости СО-параметра М ведут себя более резким образом, чем зависимости AFM-параметра $\mathrm{m}$, поэтому нами был дополнительно проведен анализ энергетических гистограмм $[24,25]$, который позволяет с высокой надежностью определить тип ФП. Для ФП I рода гистограмма распределения энергии $P(E)$ вблизи критической температуры $T_{c}$ должна иметь двухпиковую

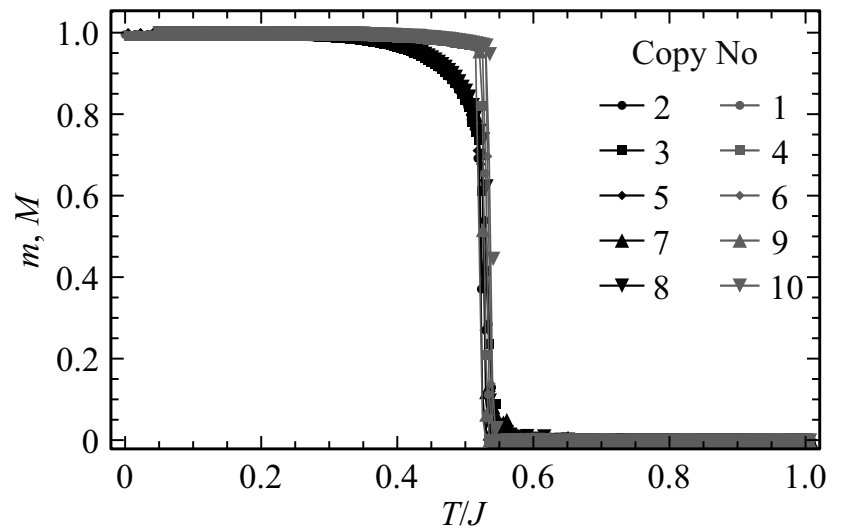

Pис. 3. Температурные зависимости параметров порядка $m$ (черные кривые) и $M$ (серые кривые) для десяти разных копий системы при $L=128, V / \tilde{J}=4, \Delta / J=1.455$. Часть копий переходит в АFM-состояние, а часть - в $\mathrm{CO}$.

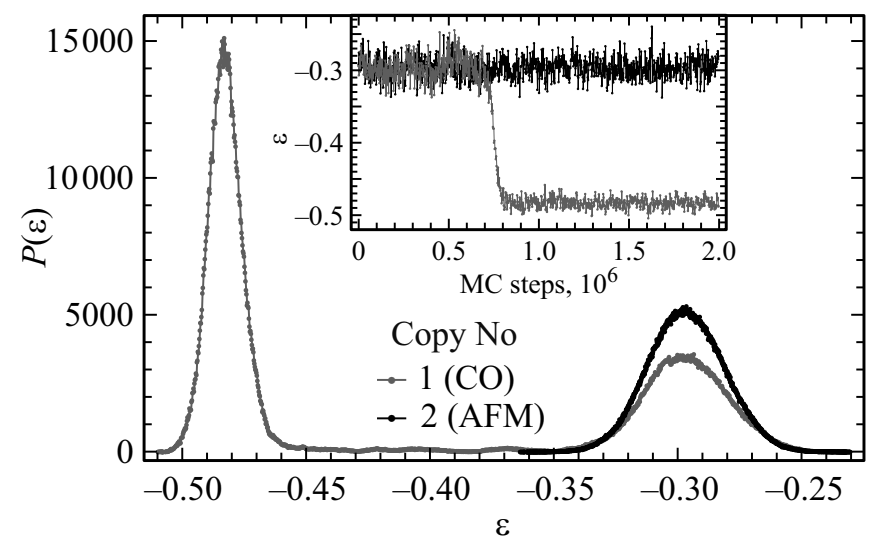

Рис. 4. Гистограммы распределения энергии $\varepsilon=E /(N J)$ для двух копий системы с параметрами $L=128, V / \tilde{J}=4$, $\Delta / J=1.455$ при температуре $T=0.5255 J$. Двухпиковая структура гистограммы для копии 1 (показана серым цветом) свидетельствует о ФП І рода в СО-состояние. Однопиковая гауссова гистограмма для копии 2 (показана черным цветом) свидетельствует о ФП ІІ рода в АFМ-состояние.

структуру, каждый пик которой соответствует определенной (стабильной или метастабильной) фазе, что отражает скачкообразное изменение энергии. В случае ФП II рода гистограмма будет иметь вид нормального распределения, где наиболее вероятное значение энергии соответствует равновесному значению энергии одной фазы при данной температуре.

На рис. 4 представлены гистограммы распределения энергии $\varepsilon=E /(N J)$ по шагам МК для копии 1 , упорядоченной зарядово, и для копии 2 , упорядоченной антиферромагнитно (см. рис. 3). Гистограммы построены вблизи критической точки при $T=0.5255 \mathrm{~J}$. Биннинг данных был произведен по 1000 интервалов для $2 \cdot 10^{6}$ шагов МК. На вставке построено распределение энергии по шагам МК. Наличие двух пиков на гистограмме распределения энергии свидетельствует о том, что переход 
в СО-состояние в случае копии 1 является ФП I рода. Пик с большей энергией соответствует неупорядоченной фазе, а с менышей - зарядовому порядку. Один хорошо выраженный максимум на гистограмме распределения энергии для копии 2 говорит о ФП II рода в AFM-состояние.

Дальнейший анализ энергетических гистограмм для различных $V / \tilde{J}$ показал, что в случае слабого спинового обмена $(V / \tilde{J}>1)$ переход в СО-состояние вблизи точки фрустрации является ФП I рода, а переход в АFMсостояние - ФП II рода. При этом ширина области с ФП І рода становится больше при увеличении соотношения $V / \tilde{J}$ и удалении от точки $V / \tilde{J}=1$. В случае сильного спинового обмена $V / \tilde{J} \leq 1$ наблюдается противоположная ситуация: переход в АFМ-состояние вблизи точки фрустрации является ФП I рода, а переход в $\mathrm{CO}-$ ФП II рода. При этом также наблюдается увеличение ширины области с ФП І рода при удалении от точки $V / \tilde{J}=1$ и уменьшении соотношения $V / \tilde{J}$.

\section{4. Заключение}

С помощью классического метода Монте-Карло проведено исследование особенностей основного состояния и фазовых переходов спин-псевдоспиновой модели двумерного магнетика с фрустрацией, вызванной конкуренцией зарядового и магнитного упорядочений. Показано, что вблизи точки фрустрации существует область, в которой вероятности формирования и зарядовой, и антиферромагнитной фаз отличны от нуля. Таким образом, одна и та же система при одинаковых условиях способна упорядочиться разным образом, т.е. основное состояние системы оказывается вырожденным не только в точке, но и в определенной области фрустрации.

Кроме того, на основе гистограммного анализа данных установлено, что фрустрация влияет на типы ФП. Вблизи точки фрустрации в случае сильного спинового обмена, для малых $V / \tilde{J}$, система испытывает ФП I рода в AFM-состояние, а в случае слабого спинового обмена, для больших $V / \tilde{J},-$ в СО-состояние.

\section{Благодарности}

Авторы благодарят А.С. Москвина за стимулирующие дискуссии.

\section{Финансирование работы}

Работа была выполнена при поддержке программы повышения конкурентоспособности Уральского Федерального университета (Акт 211 Правительства РФ, соглашение № 02.А03.21.0006 и ППК 3.1.1.1.г-20.) и Министерства образования и науки РФ, проект FEUZ-2020-0054.

\section{Конфликт интересов}

Авторы заявляют, что у них нет конфликта интересов.

\section{Список литературы}

[1] E. Fradkin, S.A. Kivelson, J.M. Tranquada. Rev. Mod. Phys. 87, 2, 457 (2015).

[2] A.S. Moskvin. Phys. Rev. B 84, 7, 075116 (2011).

[3] A.S. Moskvin. J. Phys.: Conf. Ser. 592, 1, 012076 (2015).

[4] Y.D. Panov, A.S. Moskvin, A.A. Chikov, I.L. Avvakumov. J. Supercond. Nov. Magn. 29, 4, 1077 (2016).

[5] M. Blume, V.J. Emery, R.B. Griffiths. Phys. Rev. A 4, 3, 1071 (1971).

[6] K.E. Newman, J.D. Dow. Phys. Rev. B 27, 12, 7495 (1983).

[7] J. Sivardiére, J. Lajzerowicz. J. Phys. Rev. A 11, 6, 2101 (1975); Phys. Rev. A 11, 6, 2090 (1975).

[8] C.C. Loois, G.T. Barkema, C.M. Smith. Phys. Rev. B 78, 18, 184519 (2008).

[9] S.A. Cannas, D.A. Stariolo. Phys. Rev. E 99, 4, 042137 (2019).

[10] Frustrated Spin Systems / Ed. H.T. Diep. 2nd ed. World Scientific, Singapore (2013).

[11] T.A. Kaplan, N. Menyuk. Phil. Mag. 87, 25, 3711 (2007).

[12] A. Kalz, A. Honecker. Phys. Rev. B 86, 13, 134410 (2012).

[13] L. Balents. Nature 464, 7286, 199 (2010).

[14] S.T. Bramwell, M.J.P. Gingras. Science 294, 5546, 1495 (2001).

[15] Y.D. Panov, A.S. Moskvin, A.A. Chikov, K.S. Budrin. J. Low Temp. Phys. 187, 5-6, 646 (2017).

[16] Ю.Д. Панов, В.А. Улитко, К.С. Будрин, Д.Н. Ясинская, А.А. Чиков. ФТТ 61, 5, 822 (2019).

[17] Ю.Д. Панов, А.С. Москвин, В.А. Улитко, А.А. Чиков. ФТТ 61, 9, 1676 (2019).

[18] D.N. Yasinskaya, V.A. Ulitko, A.A. Chikov, Y.D. Panov. Acta Phys. Pol. A 137, 5, 979 (2020).

[19] Д.Н. Ясинская, В.А. Улитко, Ю.Д. Панов. ФТТ 62, 9, 1543 (2020).

[20] K.S. Budrin, V.A. Ulitko, A.A. Chikov, Y.D. Panov, A.S. Moskvin. Parallel computational technologies (PCT'2018), 22 (2018).

[21] K. Binder, D.W. Heermann. Monte Carlo Simulation in Statistic̄al Physics. Springer, Berlin (1992).

[22] А.К. Муртазаев, М.К. Рамазанов, Ф.А. Кассан-Оглы, Д.Р. Курбанова. ЖЭТФ 147, 1, 127 (2015).

[23] M.K. Ramazanov, A.K. Murtazaev. Phase Transit. 91, 1, 83 (2018).

[24] F. Wang, D.P. Landau. Phys. Rev. Lett. 86, 10, 2050 (2001).

[25] A.M. Ferrenberg, D.P. Landau. Phys. Rev. B 44, 10, 5081 (1991).

Редактор Е.Ю. Флегонтова 